\title{
Analysis of atmospheric pollution by quantitative infrared thermography
}

\author{
by S.BEDNARCZYK (*), P. HERVE (*), P. ADAM (**)
}

(") Laboratoire d'Energétique et d'Economie d'Energie, Université PARIS X,1, chemin Desvallières, 92410 VILLE D'AVRAY, France.

(*) Etablissement Technique Central de l'Armement, CEB B.P.3, 91710 VERT-LE-PETIT, France.

\begin{abstract}
The radiation emitted by a cloud of particles depends on a lot of parameters: $d$, the particle diameter, $\lambda$ the wavelength, the cloud dimensions and the complex refractive index. The presence of a functional grouping of a pollutant is characterized by an absorption band whose wavelength is always in the infrared range. We have carried out a quantitative analysis of the factors governing the monochromatic emission of radiation from a cloud. The concentration, dimensions and shape of the cloud are far less significant that the particle size. For a water cloud the contrast between two wavelengths can thus vary from $-20 \%$ to $-40 \%$ according to the type of cloud, and for an oil cloud from $+10 \%$ to $+50 \%$. The comparison of two intensity levels obtained with a spectrophotometer, the first one in this range and the second one as a reference value, allows us to determine the presence/absence of a pollutant within the cloud of identical particles.
\end{abstract}

\section{Nomenclature}

T droplet temperature

d droplet diameter

a droplet ray

$n$ droplet complex refractive index

$K$ Boltzman constant

$c$ light velocity

h Planck constant

$Q$ Mie efficiency factor

$\mathrm{C}$ concentration

\author{
Greek symbols \\ $\lambda$ wavelength \\ $\varepsilon$ emissivity \\ $\alpha$ size parameter \\ $\Theta$ scattering angle \\ $\sigma$ cross section
}

\author{
Subscripts \\ abs absorption \\ diff scattering \\ ext extinction
}

\section{Introduction}

Our aim in this paper is to prove that the passive remote detection of pollutants is possible. Therefore, we have carried out in our laboratory a software computing the infrared thermal emission of liquid droplets clouds. The radiance level of a given cloud depends on its chemical composition. That 's the reason why we have purposed to compare the monochromatic thermal emission of two clouds containing two differents compounds (water and SF 98) for two wavelengths well chosen. A comparison of the contrast values for the water cloud and unknown cloud allows to determine if this unknown cloud contains SF 98 .

A NICOLET 60 SX scans the thermal emission of the cloud in the near to far infrared range. The spectrums intensity level allows to compute the bispectral contrast at 9.7 and 12 $\mu \mathrm{m}$ and shows a good agreement with the theory.

\section{Theory}

\subsection{Thermal emission}

The Planck function relates the emitted monochromatic intensity with the frequency and the temperature of an emitting medium and is expressed by :

$$
L_{\lambda}(T)=\varepsilon_{\lambda} \mathrm{L}_{\lambda}^{0}(T)=\varepsilon_{\lambda} \frac{2 h c^{2}}{\lambda^{5}\left(\exp \left(\frac{h c}{K \lambda T}\right)-1\right)}
$$




\section{http://dx.doi.org/10.21611/qirt.1992.050}

$\varepsilon_{\lambda}$ defines the monochromatic emissivity of the medium and $\mathrm{L}_{\lambda}^{0}(T)$ the black body Planck thermal emission law. In our case the medium is under the form of liquid droplets. Assuming that the droplets sizes are quite larger than the wavelength and are in thermodynamic equilibrium we may write the Kirchhoff law :

$$
\varepsilon_{\lambda}=Q_{a b s}
$$

$Q_{a b s}[1]$ represents the efficiency factor of absorption of the Mie theory, this parameter will be explicited later.

\subsection{The Mie light scattering theory [2][6][7]}

Mie has resolved the scattering of a plane wave by a homogeneous sphere. We stand here its main parameters.

Assuming that the amplitude of the incident wave is normalized to unity, the scattering intensity is given by :

$$
I(\theta)=\frac{\lambda^{2}}{4 \pi^{2} r^{2}} \frac{\left(i_{1}+i_{2}\right)}{2}
$$

$r$ represents the distance between the scattering particle and the observation point.$i_{1}$ and $i_{2}$ are called the intensity functions for the perpendicular and parallel components respectively.

$\dot{i}_{1}=\left|S_{1}\right|^{2}$, with $: \quad S_{1}(\theta)=\sum_{n=1}^{\infty} \frac{2 n+1}{n(n+1)}\left[a_{n} \pi_{n}(\cos \theta)+b_{n} \tau_{n}(\cos \theta)\right]$

$i_{2}=\left|S_{2}\right|^{2}$, with $: \quad S_{2}(\theta)=\sum_{n=1}^{\infty} \frac{2 n+1}{n(n+1)}\left[b_{n} \pi_{n}(\cos \theta)+a_{n} \tau_{n}(\cos \theta)\right]$

Note that the intensity functions depends on the the complex index of refraction, and the particle size parameter $\alpha=2 \pi a / \lambda$.

The efficiency factors of scattering $Q_{\text {diff, absorption }} Q_{a b s}$, extinction $Q_{e x t}$ define some global coefficients describing the efficiencies of the scattering absorption and extinction of the incident wave in the whole solid angle $(4 \pi)$.

$$
\begin{gathered}
Q_{\text {diff }}=\frac{2}{\alpha^{2}} \sum_{\mathrm{p}=1}^{\infty}\left(\left|\mathrm{a}_{\mathrm{p}}\right|^{2}+\left|\mathrm{b}_{\mathrm{p}}\right|^{2}\right) \\
\sigma_{e x t}=\frac{\lambda^{2}}{\pi}\{\operatorname{Re}[\mathrm{S}(0)]\} \text { et } Q_{e x t}=\frac{\sigma_{e x t}}{\pi \mathrm{a}^{2}} \\
Q_{a b s}=Q_{e x t}-Q_{\text {diff }}
\end{gathered}
$$

Note that the efficiency factors of absorption scattering and extinction are a function of the complex refractive index and the size parameter it means the particle size.

\subsection{A pattern for cloud radiation emission}

The FORTRAN software computes the radiation emission emerging from a spherical cloud.

Our program [3] consists of:

- the cloud is discretized in cubic parts involving the totality of the physical informations inside the cloud : droplets sizes distribution, number of particles per m3, water or SF 98 liquid content ;

- the Mie parameters are weighted by the size distribution in each cubic part ; 
- one cubic part is supposed to be one particle with the averaged coefficients computed in the precedent step, and emitting isotropically towards cloud surface ;

- the decrease of the radiant intensity traversing the cloud is computed for each cubic part ;

- the total thermal emission of the cloud results from the summation of each radiant intensity emerging from each cubic part.

\section{Detection of pollutants [4]}

\subsection{Contrast at two wavelengths - definition}

On using the monochromatic thermal emission values emerging out of the cloud, one can compute the contrast $\gamma$ defined by :

$$
\begin{aligned}
& \gamma_{c}=\frac{\mathrm{L}_{\lambda_{1}, T_{v}}-\mathrm{L}_{\lambda_{2}, T_{v}}}{\mathrm{~L}_{\lambda_{1}, T_{v}}+\mathrm{L}_{\lambda_{2}, T_{v}}} \\
& \gamma_{c}=\frac{Q_{a b s}\left(n_{1}, \alpha_{1}\right) \cdot L^{0}{ }_{\lambda_{1}, T_{v}} \mathrm{e}^{-\beta \operatorname{ext}\left(n_{1}, \alpha_{1}\right) z}-Q_{a b s}\left(n_{2}, \alpha_{2}\right) \cdot \mathrm{L}^{0}{ }_{\lambda_{2}, T_{v}} \mathrm{e}^{-\beta \operatorname{ext}\left(n_{2}, \alpha_{2}\right) z}}{Q_{a b s}\left(n_{1}, \alpha_{1}\right) \cdot \mathrm{L}^{0}{ }_{\lambda_{1}, T_{v}} \mathrm{e}^{-\beta \operatorname{ext}\left(n_{1}, \alpha_{1}\right) z}+Q_{a b s}\left(n_{2}, \alpha_{2}\right) \cdot \mathrm{L}^{0}{ }_{\lambda_{2}, T_{v}} \mathrm{e}^{-\beta \operatorname{ext}\left(n_{2}, \alpha_{2}\right) z}}
\end{aligned}
$$

where : $L^{0} \lambda, T_{v}$ is thePlanck blackbody thermal emission law, $\beta_{\text {ext }}$ the extinction coefficient proportionnal to Qext referred as the Beer-Bouguer-Lambert law and $\alpha=\pi \mathrm{d} / \lambda$ the size parameter.

\subsection{Theoritical results - choice of $\lambda_{1}$ and $\lambda_{2}$}

As it is said in introduction, our study concerns water cloud and SF 98 cloud. As many organic compounds SF 98 has a typical peak of absorptivity at $9.7 \mu \mathrm{m}\left(1030 \mathrm{~cm}^{-1}\right)$. So the first wavelength $\lambda_{1}$ is imposed at $9.7 \mu \mathrm{m}$. To choice $\lambda_{2}$ we have computed the contrast between $\lambda_{1}$ and a large group of $\lambda_{2}$ values in the $8-12 \mu \mathrm{m}$ range.

\section{. Case of SF 98 clouds}

The ETCA CEB has given us the complex refractive index of SF 98.

The parameters on wich can depend the contrast are : the droplets size, the compound liquid content, the cloud size.

Figures 1,2 and 3 show the predicted results. As we can see on the following figure, only the size distribution has a big influence on the contrast values.

\section{. Comparison with a water cloud}

The Querry's article [5] has given us the complex refractive index of water.

The figure 4 shows the contrast values for a water cloud containing droplets having a diameter bigger than 6 microns.

The water and SF 98 droplets diameter have been chosen in order to approach ourself as much as possible from the diameters generated in laboratory and in order to be in good agreement with the atmospheric clouds size distribution [8][9]. In real cases the SF6 droplets sizes are around $200 \mu \mathrm{m}$ and the water droplets sizes are less than $40 \mu \mathrm{m}$.

The figure 5 shows that the biggest contrast difference between the two compounds appear when $\lambda_{2}$ is equal to $12 \mu \mathrm{m}$. 
http://dx.doi.org/10.21611/qirt.1992.050

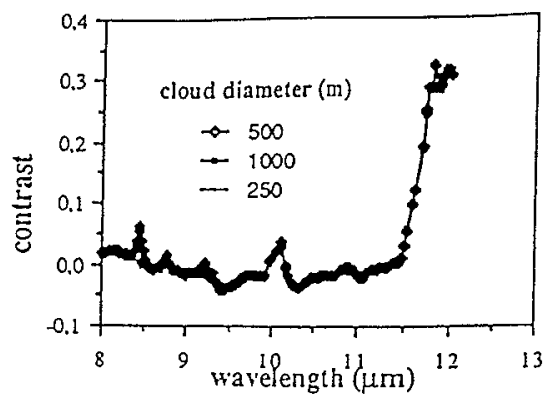

Fig.1. - Contrast around $9.7 \mu \mathrm{m}$ for three different clouds diameters

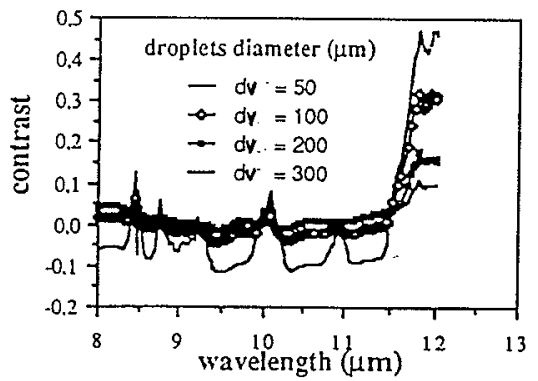

Fig.3. - Contrast around $9.7 \mu \mathrm{m}$ for three different droplets diameters

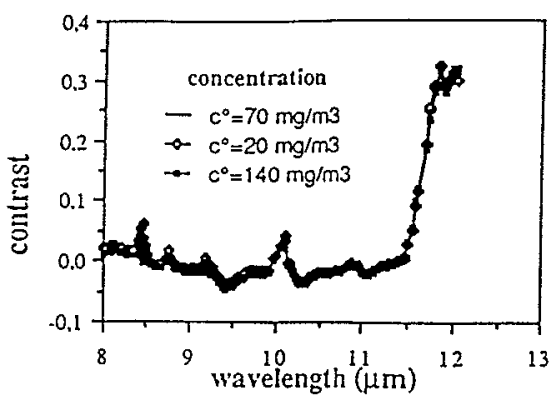

Fig.2. - Contrast around $9.7 \mu \mathrm{m}$ for three differents liquid contents

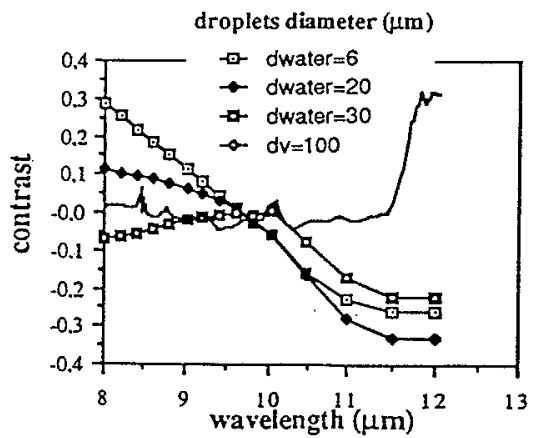

Fig.4. - Comparison between the contrast around $9.7 \mu \mathrm{m}$ for water and SF98

So we choose $12 \mu \mathrm{m}$ for $\lambda 2$ and for our future experiments.

Note that at $9.7 \mu \mathrm{m}$ the complex refractive index for SF6 is $n=1.462-i 0.2596$ and $n=1$. $111-i 0.008$ at $12 \mu \mathrm{m}$. For water $\mathrm{n}=1.239-i 0.045$ at $9.7 \mu \mathrm{m}$ and

$\mathrm{n}=1.444-\mathrm{i} 0.199$ at $12 \mu \mathrm{m}$.

\section{- Temperature effect}

The figures 5 and 6 contain curves plotting the contrast values between 9.7 and $12 \mu \mathrm{m}$ for each compounds.

In the two cases the contrast arises linearly with the temperature. When this one increases from 273 to $298 \mathrm{~K}$ the contrast given by the two clouds put $4 \%$ up.

$\mathrm{A} \Delta \mathrm{T}$ of $5 \mathrm{~K}$ corresponds to a $\Delta \gamma$ of $4 \%$.

\section{- Validation of the thermal emission}

In order to valid the thermal emission pattern it is necessary to take into account the grey emittance of the earth surface and the black emittance of the sun (figure 7 )wich is scattered by the cloud droplets. We have obtained $10^{-4}-10-8$ for the scattering intensity and thermal emission ratio. The thermal emission pattern is justified. 
http://dx.doi.org/10.21611/qirt.1992.050

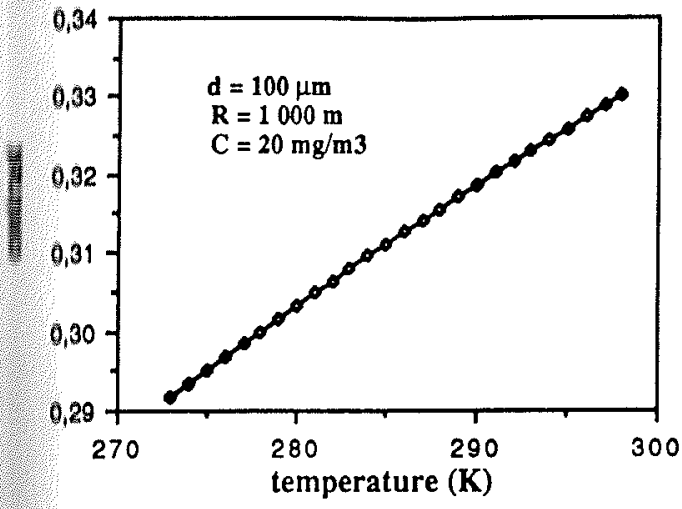

IIg.5. - Contrast around $9.7 \mu \mathrm{m}$ against temperature for water cloud

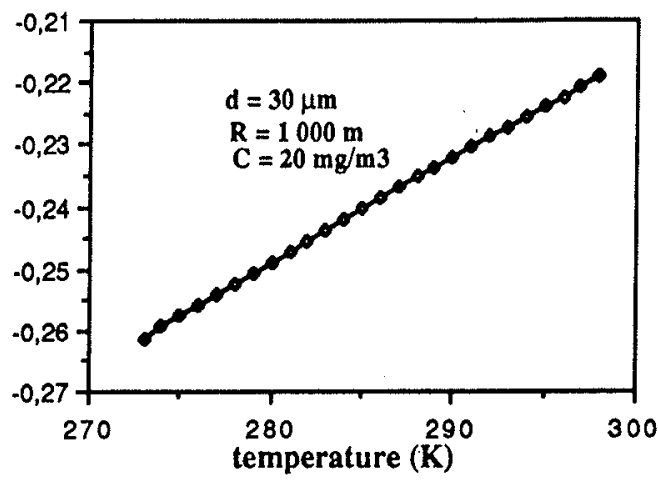

Fig.6. - Contrast around $9.7 \mu \mathrm{m}$ against temperature for SF98 cloud

\section{Experiments and results}

\subsection{Experiments}

The T.F. spectrophotometer NICOLET $60 \mathrm{SX}$ allows us to realize a near to far infrared epectrums. The following scheme (figure 8 ) represents the experimental set-up mesuring the thermal emission of a liquid droplets cloud.

The $\mathrm{HgCdTe}$ detector owns a $10^{11-12}$ detectivity $\left(\mathrm{D}^{*}\right)$. Its maximal sensivity is around 12.5 um.

The water cloud comes out from an EVIAN spray wich generates around $40 \mu \mathrm{m}$ droplets. The SF6 clouds comes out from a spray sailed in the commerce and generating droplets bigger than $200 \mu \mathrm{m}$.

We didn't succeed in the determination of liquid content inside the cloud.

The system parameters is the following one

- resolution : $8 \mathrm{~cm}^{-1}$

- gain : 20

- averaging on 64 consecutive spectrums

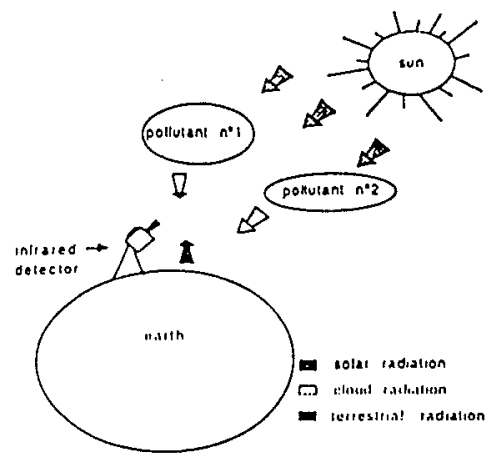

Fig.7. - Geographic context

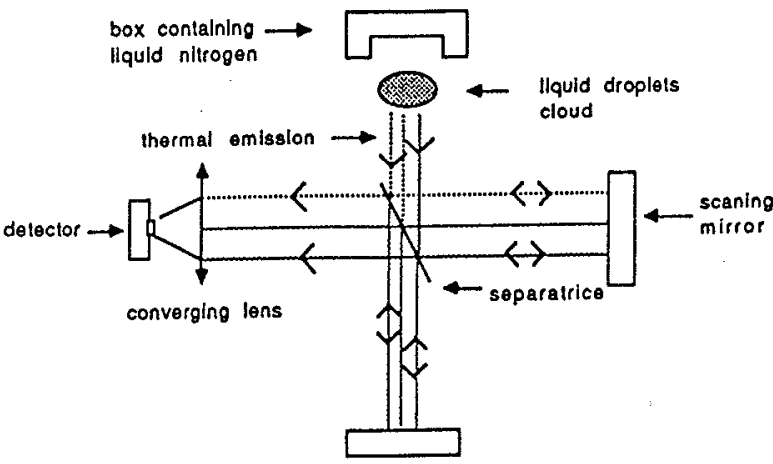

Fig.8. - Experimental set-up 


\section{http://dx.doi.org/10.21611/qirt.1992.050}

We express our gratitude to P. MASCLET (ETCA Arcueil) for doing the experiments.

The first experiment consists of measure the general experimenal set-up background it means the room and the spectrophotometer (figure 9). Note that the intensity scale is arbitrary.

In order to quantify the experiment noise and to compute the measures incertitudes we plot the two averaged spectrums ratio (figure 10).

The figure 9 shows that manipulation noise is constant in the $9-15 \mu \mathrm{m}$ spectral range. There is $5 \%$ of measure incertitude for a 64 spectrums averaged spectrum.

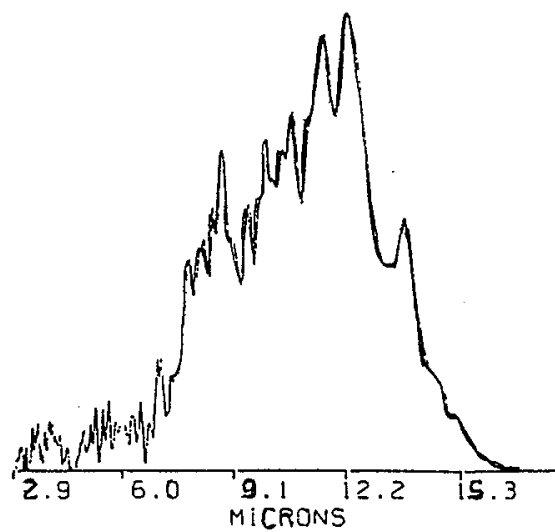

Fig.9. - Experiment background level

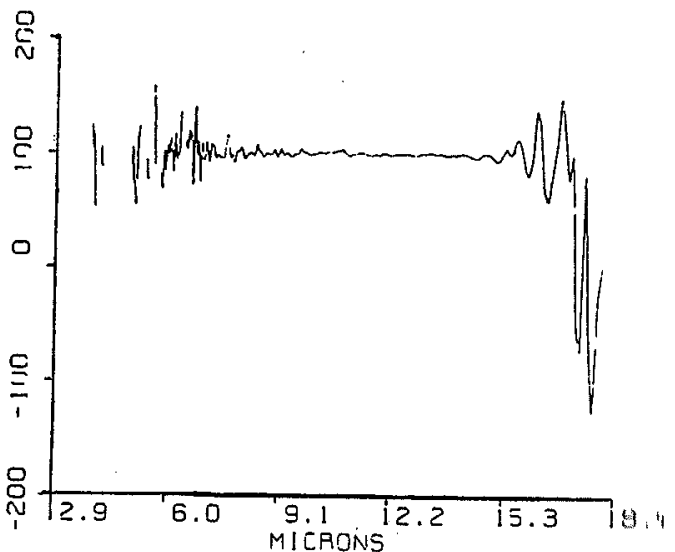

Fig.10. - Two averaged background spectrums ratlo

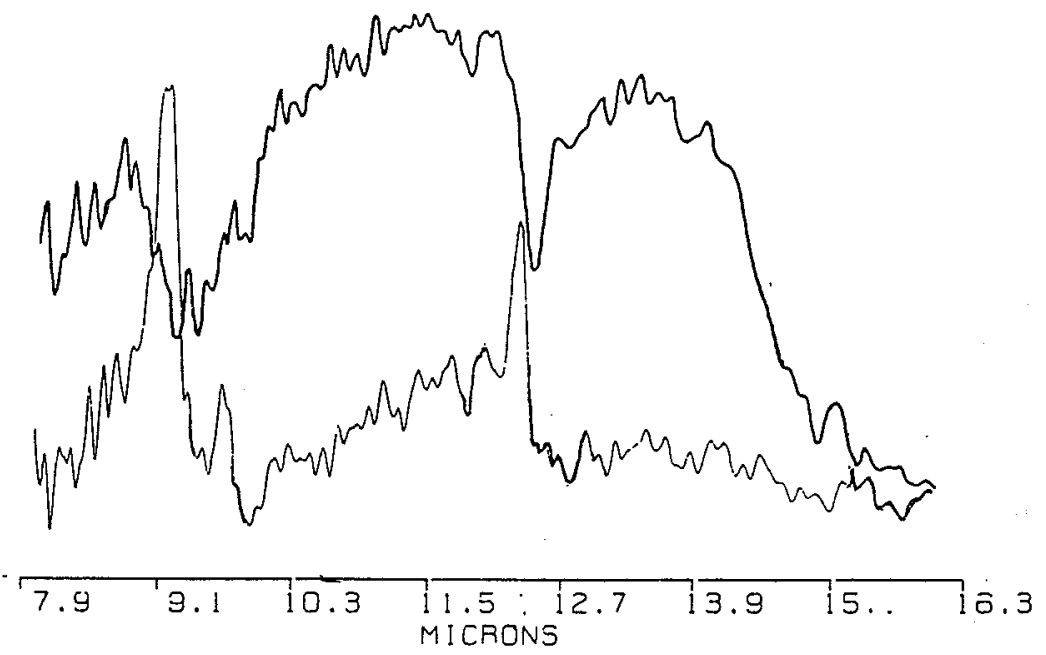

Fig.11. Water (black curve) and SF6 (grey curve) liquid droplets spectrums 


\section{http://dx.doi.org/10.21611/qirt.1992.050}

Each spectrum intensity scales were obviously the same. The following array contains the signals dynamic a and $b$ for each wavelength 9.7 and $12 \mu \mathrm{m}$ (respectively) :

\begin{tabular}{|c|c|c|}
\hline wavelength (micr.) & 9.7 & 12 \\
\hline water & $\mathrm{a}=6.29$ & $\mathrm{~b}=11.25$ \\
\hline SF6 & $\mathrm{a}=3.87$ & $\mathrm{~b}=2.24$ \\
\hline
\end{tabular}

Therefore we can compute the contrast values for each cloud:

\begin{tabular}{|l|c|c|}
\hline & water & SF6 \\
\hline contrast $=(a-b) /(a+b)$ & $-27.8 \% \pm 0.7 \%$ & $+27 \% \pm 2 \%$ \\
\hline
\end{tabular}

\subsection{Results}

The following array resume the experimental and theoritical contrast values.

\begin{tabular}{|l|l|l|}
\hline & \multicolumn{1}{|c|}{ water } & \multicolumn{1}{|c|}{ SF6 } \\
\hline diameter range (micr.) & 6 to30 & 100 to 300 \\
\hline $\begin{array}{l}\text { theoritical contrast } \\
\text { range }\end{array}$ & $-35 \%$ to $-20 \%$ & $10 \%$ to $30 \%$ \\
\hline experimental diameter & 20 to 50 & $>200$ \\
\hline experimental contrast & $-27.8 \%$ & $+27 \%$ \\
\hline
\end{tabular}

The contrast values between 9.7 and $12 \mu \mathrm{m}$ seem to be in good agreement with the theory.

\section{Conclusion}

The possibility of a passive remote detection of pollutants in the case of liquid droplets clouds has been proved. The bispectral contrast seems to be a good way for the passive remote detection if the two wavelengths are well choosen.

\section{REFERENCES}

1. C.F BOHREN, How can a particle absorb more than the light incident on it ? Journal of Physics, Vol. 51 (4), pp. 323-327, April 1983.

2. M. KERKER, The scattering of light , Academic Press, 1969.

3. S. BEDNARCZYK, P. HERVÉ, P. ADAM, Analyse par thermographie infrarouge de la granulométrie des nuages, S.F.T. , 06.12.1991, Thermographie Infrarouge Quantitative.

4. D. F. FLANIGAN, Detection of organic vapors with active and passive sensors: a comparison, Applied Optics, Vol 25 (23), December 1986.

5. G.M HALE, M.R QUERRY, Optical Constants of Water in the $200 \mathrm{~nm}$ to $200 \mu \mathrm{m}$ Wavelength Region, Applied Optics, 12, 555-562, 1973.

6. C. F BOHREN, D.R HUFFMAN, Absorption and scattering of light by small particles, J. Wiley and Sons, 1983.

7. KUO-NAN LIOU, An introduction to atmospheric radiation, Academic Press, 1980.

8. A.C BEST, Drop - size distribution in cloud and fog, 1951.

9. D. DEIRMENDJIAN, Electromagnetic scattering of spherical polydispersions, Elsevier, 1969. 